\title{
Androgen Receptors: A Marker to Increase Sensitivity for Identifying Breast Cancer in Skin Metastasis of Unknown Primary Site
}

Ilene B. Bayer-Garner, M.D., Bruce Smoller, M.D.

Departments of Pathology (IBB-G, BS) and Dermatology (BS), The University of Arkansas for Medical

Sciences, Little Rock, Arkansas

\begin{abstract}
Metastatic lesions to the skin may present a dilemma in the identification of the primary site. Breast carcinoma, metastatic to the skin, that is negative for estrogen receptors (ERs) and/or progesterone receptors (PRs) may be mimicked by a number of other metastatic lesions. In the present study, 16 formalin-fixed and paraffin-embedded infiltrating ductal carcinomas metastatic to the skin, which were $\mathrm{ER}-/ \mathrm{PR}-$, ER-/PR+, or ER+/PR-; 5 metastatic lesions to the skin from primary lesions other than breast cancer; and 5 eccrine tumors were examined for immunoreactivity to the androgen receptor. The majority of the metastatic breast lesions (82\%) exhibited immunopositivity for androgen receptor, whereas the metastatic skin lesions from primary lesions other than breast cancer and the eccrine tumors were immunonegative. Thus, androgen receptor immunohistochemistry could serve as a marker to increase sensitivity for identifying breast cancer in skin metastasis of unknown primary sites.
\end{abstract}

KEY WORDS: Androgen receptor, Breast cancer metastases to the skin, Estrogen receptor, Progesterone receptor.

Mod Pathol 2000;13(2):119-122

In the examination of metastatic tumors to the skin, the identification of the site of the primary tumor is of importance as some carcinomas first come to the attention of the pathologist as skin metastases. Schwartz (1) found that $7.6 \%$ of carcinomas were first identified as skin metastases. In breast cancer metastases, presence of estrogen receptors (ERs)

Copyright () 2000 by The United States and Canadian Academy of Pathology, Inc.

VOL. 13, NO. 2, P. 119, 2000 Printed in the U.S.A.

Date of acceptance: September 10, 1999.

Address reprint requests to: Bruce R. Smoller, M.D., Professor of Pathology and Dermatology, Department of Pathology, Slot 517, The University of Arkansas for Medical Sciences, 4301 West Markham Street, Little Rock, AR 72205. and progesterone receptors (PRs), identified by immunohistochemical stains, may aid in the identification of the primary site; however, eccrine neoplasms have also been shown to express ERs and PRs (2). Are there any additional clues to help one narrow the diagnosis to that of a metastatic breast lesion? It has been reported that in primary breast tumors, between $80 \%(3,4)$ and $100 \%(5)$ of lesions are androgen receptor (AR) positive and $60 \%$ are positive for ARs, ERs, and PRs (6), whereas $25 \%$ of metastatic breast lesions are solely AR+ (7). ER, PR, and AR concentrations are lower in metastases than in the primary tumors $(7,8)$; of the three, ARs seem to be best preserved in metastases (3). Little is known about the role and clinical significance of AR in the biologic behavior of breast cancer (3), and even less is known about AR status in breast carcinomas that are metastatic to the skin. The discovery of a new antibody corresponding to amino acids 299 through 315 of the human AR presented the opportunity to assess the immunoreactivity of breast cancer metastases to the skin. Accordingly, this study was undertaken to assess the AR status of these neoplasms and to determine whether AR immunohistochemistry could serve as a marker to increase sensitivity for identifying breast cancer in skin metastasis of unknown primary site.

\section{MATERIALS AND METHODS}

A total of 21 biopsies of carcinomas, metastatic to the skin, and 5 primary eccrine tumors were obtained from the pathology tissue files at the University of Arkansas for Medical Sciences. The neoplasms were 16 metastatic infiltrating ductal breast carcinomas; 2 metastatic squamous cell carcinomas; 1 metastatic gastric carcinoma; 1 metastatic ovarian carcinoma; 1 metastatic melanoma; 1 eccrine acrospiroma; 2 eccrine poromas; and 2 syringomas, 1 of which was the clear cell variant. The specimens were fixed in $10 \%$ formalin and embedded in paraffin. Four-micron-thick sections were 
cut and mounted on 3-aminopropyltrethoxy-silane-coated slides, dried, and deparaffinized before undergoing antigen retrieval by heat treatment in DAKO Target Retrieval solution (DAKO, Carpenteria, CA). The endogenous peroxidase activity was quenched with prediluted peroxidase block, and nonspecific binding was quenched with horse serum block. The Immunocruz Staining System @ (Santa Cruz Biotechnology Inc., Santa Cruz, CA) was used with AR (441) as the primary prediluted antibody, a mouse monoclonal IgG $_{1}$ antibody corresponding to amino acids 299 through 315 of the human AR. Sections were incubated for $2 \mathrm{~h}$ with the primary antibody and subsequently incubated with the biotinylated secondary antibody for $30 \mathrm{~min}$. The signal was visualized with $3,3^{\prime}$-diaminobenzidine in chromogen solution with Imidazole- $\mathrm{HCl}$ buffer at pH 7.5 (DAKO liquid 3,3' -diaminobenzidine + large volume substrate-chromogen system), yielding a brown end product at the site of the target antigen. AR positivity was defined as strong nuclear binding in greater than $90 \%$ of the cells in question. Prostate carcinoma tissue served as the positive control.

\section{RESULTS}

Twenty-one carcinomas, metastatic to the skin, and 5 primary eccrine skin tumors were analyzed for immunoreactivity to the AR. The 16 breast carcinomas had been previously analyzed for immunoreactivity to both ERs and PRs. These tissue samples consisted of 11 lesions that were immunonegative to both ERs and PRs, 3 lesions that were estrogen immunopositive but progesterone immunonegative, and 2 lesions that were progesterone immunopositive but estrogen immunonegative. All of the metastatic breast carcinomas were identified as the infiltrating ductal type, and of those that had received a Bloom-Richardson grade, the majority were high-grade lesions. Thirteen of the primary breast lesions had been analyzed for immu- noreactivity to ER and PR. Of these 13 lesions, 3 showed a discrepancy in the ER/PR immunoreactivity between the breast primary and the skin metastasis. One primary breast lesion was $\mathrm{ER}+/ \mathrm{PR}+$, whereas the skin metastasis was $\mathrm{ER}-/ \mathrm{PR}-$. Another breast primary lesion was ER-/PR-, whereas the skin metastasis was $\mathrm{ER}-/ \mathrm{PR}+$. The final breast primary lesion showing a discrepancy in ER/PR immunoreactivity from the skin metastasis was $\mathrm{ER}+/ \mathrm{PR}+$, whereas the skin metastasis was $\mathrm{ER}-/ \mathrm{PR}+$ (Table 1).

Positive nuclear AR antibody immunohistochemical staining was observed in the majority of metastatic breast carcinomas (Fig. 1A), with 11 of 16 showing immunonegativity for both ERs (Fig. 1B) and PRs (Fig. 1C). The metastatic lesions other than those metastatic from the breast, namely, the metastatic squamous cell carcinoma, gastric carcinoma, ovarian carcinoma, and melanoma, showed uniform immunonegativity for the AR. The eccrine neoplasms all showed uniform AR negativity with AR immunopositivity evident within normal sebaceous glands. Of the 11 metastatic breast lesions that were ER-PR-, 9 (82\%) exhibited nuclear immunopositivity for the AR. The five lesions that were either ER-/PR+ or ER+/PR- all exhibited nuclear AR immunopositivity. Staining within all tumors was heterogeneous; some nuclei exhibited immunopositivity, whereas others did not. The proportion of tumor cell nuclei exhibiting immunopositivity was in the range of $90 \%$. The metastatic lesions other than breast cancer metastases, which bear some morphologic similarity to breast cancer metastases, all showed AR immunonegativity as did all of the eccrine neoplasms.

\section{DISCUSSION}

We have shown that AR immunoreactivity may be a marker to increase sensitivity for metastatic breast cancer in the identification of an unknown

TABLE 1. Breast Cancer Metastases to the Skin with Androgen, Estrogen, and Progesterone Immunoreactivity: Comparison to Primary Breast Sex Steroid Immunoreactivity

\begin{tabular}{|c|c|c|c|c|c|}
\hline Skin Metastasis AR & Skin ER & Skin PR & Bloom-Richardson Grade & Breast ER & Breast PR \\
\hline pos & pos & neg & & & \\
\hline pos & neg & neg & III/III & neg & neg \\
\hline pos & neg & neg & III/III & neg & neg \\
\hline pos & neg & neg & III/III & neg & neg \\
\hline pos & neg & neg & III/III & pos & pos \\
\hline pos & pos & neg & II/III & pos & neg \\
\hline pos & neg & pos & & neg & neg \\
\hline pos & neg & pos & & pos & pos \\
\hline pos & pos & neg & III/III & pos & pos \\
\hline pos & neg & neg & & & \\
\hline pos & neg & neg & III/III & neg & neg \\
\hline pos & neg & neg & & & \\
\hline pos & neg & neg & & & \\
\hline pos & neg & neg & III/III & neg & neg \\
\hline neg & neg & neg & & neg & neg \\
\hline neg & neg & neg & & neg & neg \\
\hline
\end{tabular}

AR, androgen receptor; ER, estrogen receptor; PR, progesterone receptor; pos, positive; neg, negative. 

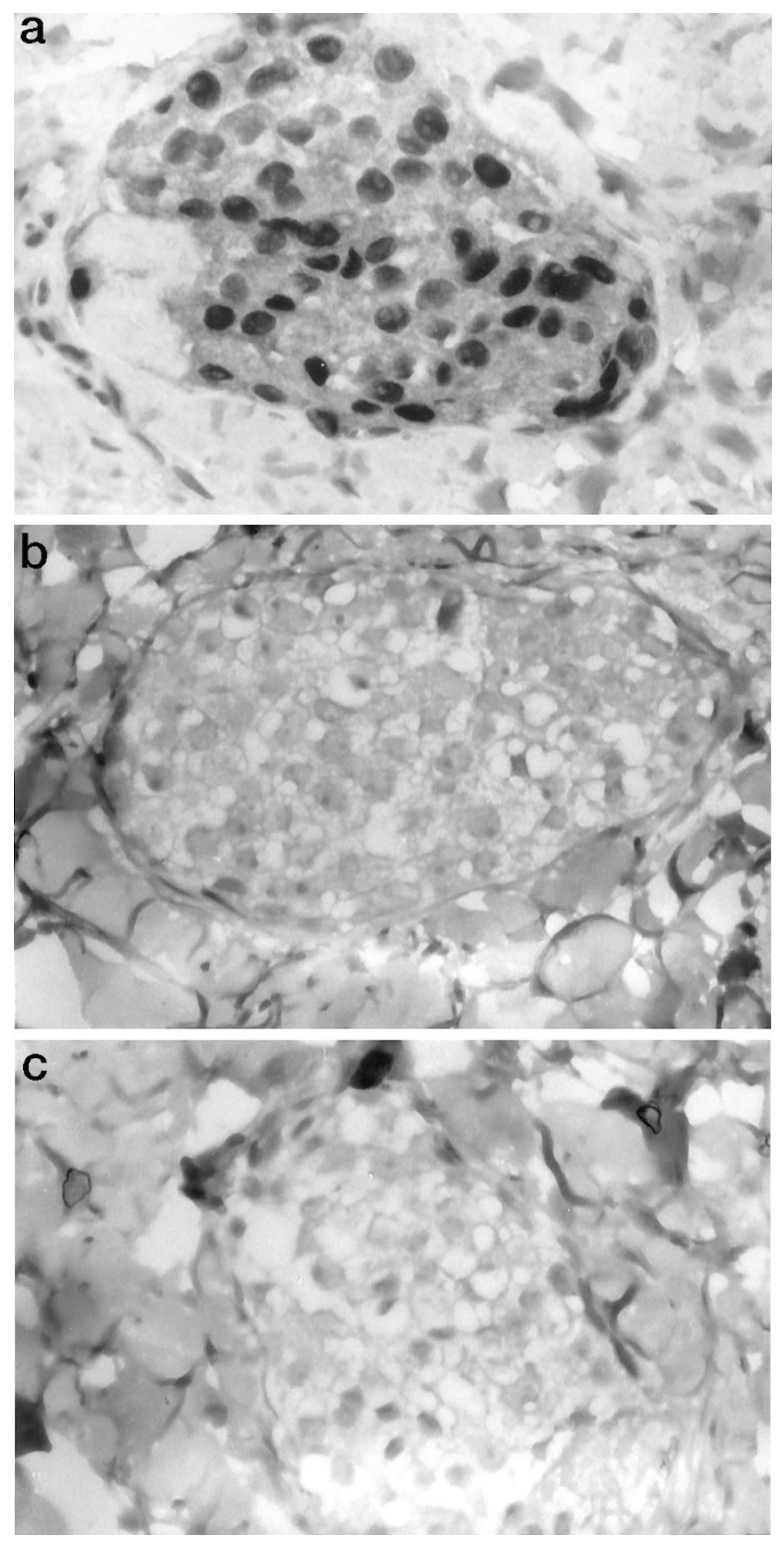

FIGURE 1. A, androgen receptor immunopositivity in infiltrating ductal breast carcinoma metastatic to the skin. There is strong nuclear androgen receptor staining. B, estrogen receptor

immunohistochemistry of the same lesion as shown in Figure 1A. Note the lack of nuclear immunoreactivity for the estrogen receptor. C, progesterone immunohistochemistry of the same lesion as shown in Figure 1A. Note the lack of nuclear immunoreactivity for the progesterone receptor.

primary skin metastasis. Our results show that the majority of metastatic breast carcinomas that are negative for both ERs and PRs or that exhibit immunonegativity for either ERs or PRs show nuclear immunopositivity for the AR. Metastatic lesions that may mimic breast metastases, namely, squamous cell carcinoma, gastric carcinoma, ovarian carcinoma, and melanoma, showed uniform immunonegativity for the AR. Eccrine neoplasms, which may show immunopositivity for ERs or PRs (2), showed uniform AR immunonegativity. There was a difference in the ER/PR status between the breast primary lesion and the skin metastasis in 3 of 13 tumors.
The majority (87\%) of invasive ductal breast carcinoma, metastatic to the skin, that are ER-/PR-, $\mathrm{ER}+/ \mathrm{PR}-$, or ER-/PR+ show immunoreactivity for ARs. This could be advantageous in the differentiation of these tumors from other metastatic lesions that can mimic breast cancer metastases. There were, however, 2 of 11 skin metastases (18\%) that were $\mathrm{ER}-/ \mathrm{PR}-$ as well as AR-. It is in these few cases that AR immunohistochemistry cannot aid in the identification of an unknown primary site.

A difference was found in the ER/PR status between the breast primary lesion and the skin metastasis in 3 of 13 tumors. In two cases, there was a loss of sex steroid expression in the metastasis, whereas the third case showed a gain of PR immunopositivity. Loss of sex steroid expression in metastatic lesions has been previously noted. ER, PR, and AR concentrations are lower in metastases than in the primary tumors $(7,8)$. The single case with gain of PR is an anomaly that might be explained by presence of more than one primary breast lesion or by mutation of part of a single lesion from an ER-I $\mathrm{PR}+$ tumor, which metastasized, to an ER-/PRtumor that was sampled for receptor status.

Cutaneous metastases from primary tumors other than breast that may mimic breast cancer metastases were also examined for immunoreactivity to the AR. These were found to be nonreactive. There are additional primary carcinomas that are known to contain hormone receptors that metastasize to the skin, namely, thyroid, hepatocellular, and genital cancers. However, these carcinomas are rare and were not examined in the current study because of the inability to locate any examples of these in tissue files.

In conclusion, we have shown that AR immunoreactivity may be a marker to increase sensitivity for metastatic breast cancer in the identification of an unknown primary skin metastasis that is ER-/PR-, $\mathrm{ER}+/ \mathrm{PR}-$, or $\mathrm{ER}-/ \mathrm{PR}+$. Cutaneous metastatic disease is the initial presentation in $7.6 \%$ of patients (1); thus, it is important to identify the site of the primary tumor whether it is for diagnostic, prognostic, or therapeutic utility. When a metastatic lesion of unknown primary site is encountered in the skin, the immunohistochemical analysis for possible site of origin should include a minimum of cytokeratin, ERs, and PRs. It is our hypothesis that the addition of AR immunohistochemistry may increase the diagnostic yield in identification of those metastatic breast lesions that are not $\mathrm{ER}+/ \mathrm{PR}+$.

\section{REFERENCES}

1. Schwartz RA. Cutaneous metastatic disease. J Am Acad Dermatol 1995;33:161-82.

2. Wallace ML, Smoller BR. Estrogen and progesterone receptors and anti-gross cystic disease fluid protein 15 (BRST-2) 
fail to distinguish metastatic breast cancer from eccrine neoplasms. Mod Pathol 1995;8:897-901.

3. Kuenen-Boumeester V, Van der Kwast TH, van Putten WLJ, Claassen C, van Ooijen B, Henzen-Logmans SC. Immunohistochemical determination of androgen receptors in relation to oestrogen and progesterone receptors in female breast cancer. Int J Cancer 1992;52:581-4.

4. Isola JJ. Immunohistochemical demonstration of androgen receptor in breast cancer and its relationship to other prognostic factors. J Pathol 1993;170:31-5.

5. Hall RE, Aspinall JO, Horsfall DJ, Birrell SN, Bentel JM, Sutherland RL, et al. Expression of the androgen receptor and an androgen-responsive protein, apolipoprotein D, in human breast cancer. Br J Cancer 1996;74:1175-80.

6. Kuenen-Boumeester V, Van der Kwast TH, Claassen CC, Look MP, Liem GS, Klijn JG, et al. The clinical significance of androgen receptors in breast cancer and their relation to histological and cell biological parameters. Eur J Cancer 1996;32A:1560-5.

7. Lea OA, Kvinnsland S, Thorsen T. Improved measurement of androgen receptors in human breast cancer. Cancer Res 1989;49:7162-7.

8. Mercer RJ, Lie TH, Rennie GC, Bennett RC, Morgan FJ. Hormone-receptor assays in breast cancer: a five-year experience. Med J Australia 1983;1:365-9.

\section{Book Review}

\section{Perry DJ, Pasi KJ: Hemostasis and Thrombosis Protocols, 368 pp, Totowa, New Jersey, Humana Press, Inc., 1999 (\$89.50).}

This is a reference manual on research and diagnostic techniques in the field of hemostasis and thrombosis. It is written by multiple experts from the United Kingdom, the United States, and Australia. The majority of the text is devoted to contemporary nucleic acid approaches, but some sections also describe techniques for protein and cellular expression analysis.

The manual is divided into five parts. Part one is an introductory chapter that briefly summarizes the fundamental components of the hemostatic mechanism. Part two is a series of chapters that describe basic nucleic acid techniques that are applied in later chapters, such as the isolation, amplification, and sequencing of nucleic acids, as well as various methods for analyzing promoter regions. Part three, as well as some chapters within part four, discuss different screening methods for detecting genetic mutations, which can be applied to any gene. Part four contains numerous chapters that describe various nucleic acid, cellular expression, and protein-based methods that have been applied to analyze specific clotting factor deficiencies and hypercoagulable states. Part five discusses methods for the molecular biological analysis of platelet and megakaryocyte disorders.

Each chapter is presented in a recipe format so that the methods can be reproduced. All chapters are subdivided into sections for easy reading: introduction, materials, methods, and notes. The "notes" section provides useful caveats about experimental technique and data interpretation. The chapters on mutation detection in clotting factor deficiencies will be useful to hemostasis laboratories that are interested in carrier detection, prenatal diagnosis, or research. The chapters on von Willebrand factor multimer analysis, factor $\mathrm{V}$ Leiden mutation, and prothrombin G20210A mutation will be useful to hemostasis laboratories that want to set up these important diagnostic tests.

The criticisms are relatively minor. One of the mutation detection methods (chemical cleavage of mismatched base pairs) is first introduced in part four but would have been more appropriate in part three to provide consistency. The text would have benefited from careful editing to correct typographical errors found in several chapters. Some chapters lacked pictures of electrophoretic gels, which if present would have enhanced understanding of the material.

This manual does well at meeting its goals of providing a broad overview of techniques used in the analysis of hemostatic disorders and providing strategies for future problem solving. It will be of great utility to hemostasis laboratories that wish to advance their research or diagnostic capabilities.

\section{Mark Cunningham \\ University of Kansas School of Medicine \\ Kansas City, Kansas}

